\title{
A Plant Growth Simulation Algorithm for solving nonlinear equation group
}

\author{
Zhengen Fan ${ }^{1, \mathrm{a}}$ Yueguang $\mathrm{Li}^{2, \mathrm{~b}}$ \\ 1 Gansu Normal University for Nationalities, Hezuo, gansu, 747000, \\ China \\ 2Gansu Normal University for Nationalities, Hezuo, gansu, 747000, \\ China \\ Yaeguangli7@163.com, ${ }^{a}$ Yueguangli7@sohu.com
}

\begin{abstract}
In this paper, according to the problems such as high sensitivity to the initial guess of the solution and poor convergence reliability of the classical algorithms used to solve nonlinear equations, a Plant Growth Simulation Algorithm(PGSA) was put forward, and it was used to solve the systems of nonlinear equations, the algorithm was experimented and the experimental results show that the new algorithm has reliable convergence, high convergence rate and solution precision.

Keywords: Plant Growth Simulation Algorithm(PGSA); Nonlinear equations; function; Intelligent algorithm
\end{abstract}

\section{Introduction}

The equations is one of the basic problems in numerical linear algebra, many engineering application and scientific computing problems require the solution of equations finally, therefore, studying the equations has important significance $^{[1]}$. In the traditional method mainly has the Newton method ${ }^{[2]}$, iterative method, gradient method, evolutionary algorithms such as PSO algorithm $^{[3,4]}$. The traditional method has high accuracy, but the equations with high performance requirements, such as the requirements of continuous and differentiable equations. Evolutionary algorithm can improve the population size and the number of iterations to improve accuracy, the solving time is proportional to iterative times, time efficiency decreased obviously. 


\section{The plant growth simulation algorithm}

Plant growth simulation algorithm(PGSA) is an evolutionary computation technique through simulating the growth processes in plants, which is based on the principles of plant phototropism growth ${ }^{[5]}$. The algorithm has a good prospect in the application due to requiring a simple on the parameters. It has gradually been applied in the field of engineering by many scholars ${ }^{[6]}$.

Plants can be viewed as a system, which composes of a large number of branches and nodes. It must be as soon as possible to strive for breeding more branches and leaves for earning more surface areas, which can obtain the greatest possible sunlight. The form of grammar that simulates the plant description analysis and development had been established since linguistics was introduced into the biological, which based on a simple rewrite rules and branching rules, this is called L-system. Formal description of plant growth can be carried out as the following:

1. The section of the site grow new branches which first emerged in a number of stems is called the growth node.

2. Most of the new branches have grown updated branches, and the process repeated on the old and new branches.

3. Different branches in the tree have similarities with each other, and the entire plant has self-similar structure.

The Branching model of plant growth is improved according to L-system combined with computer graphics and fractal theory, the plant, as the form of plant growth characteristics, can be described as follow: Supposed branches of plant growth occurred in the two-dimensional plane, Each branch growing in units length every time, or rotating a certain angle $\alpha$, Starting from the node of trunk or branches, The growth process were repeated through rewriting the rules of branches of plant growth in the two dimensional plane.

It was proved by biological experiments that a new branch that is able to grow depends on its morphactin concentration when the plant has more than one node. The node which has larger value of morpheme concentration has more 
growth opportunities than the smaller one, morphactin concentration are not pre-assigned to the nodes, but formed according to their location information which we call that plant showed the feature of plant's Phototropism. After the node has been formed, morphactin concentration will be readjusted according to changing in the environment of a new growth node else.

We could describe the characteristics of plant growth in term of the mathematical view basis on plant growth analysis of the above. Suppose the length of tree trunk is $\mathrm{T}$, the length of branch is $\mathrm{L}$, there are $\mathrm{W}$ growing nodes $S_{T}=\left(S_{T 1}, S_{T 2}, \ldots, S_{T W}\right)$ in the trunk, morphactin concentration of those nodes is $P_{T}=\left(P_{T 1}, P_{T 2}, \ldots, P_{T W}\right) \quad$ respectively. There are $q$ growth nodes $S_{T}=\left(S_{T 1}, S_{T 2}, \ldots, S_{T q}\right)$ in the branch. Morphactin concentration of those nodes is $P_{T}=\left(P_{T 1}, P_{T 2}, \ldots, P q\right)$ respectively. The morphactin concentration which grown in the trunk and branch is calculated as follows:

$$
P_{T i}=f\left(x_{0}\right)-f\left(S_{T i}\right) / \sum_{i}^{W}\left(f\left(x_{0}\right)-f\left(S_{T i}\right)\right)+\sum_{j}^{q}\left(f\left(x_{0}\right)-f\left(x_{T q}\right)\right)
$$

Where $x_{0}$ represents the root of plant (the initial node), $f(*)$ is the information function of the node in the environment, The smaller value of the function as possible shows the better environment where the node located, it will help to grow new branch.

From formula (1), the value of morphactin concentration in every node depends on relative position of the relative to the initial root and the location of the environmental information, the mechanism consistent with the formation of the morphactin concentration in the plant cell. We can deduced from formula (1) as follows:

$$
\sum_{i=1}^{T} \sum_{j=1}^{q}\left(P_{T i}+P_{T j}\right)=1
$$


We can produce random number in the interval $[0,1]$. The random number must fall within one of the state space $\left(P_{1}, P_{2}, \ldots, P_{T+q}\right)$, where the corresponding node will have a prior right to grow new branch. A simple example of morphactin concentration state space displays in the fig. 1.The value of morphactin concentration in all nodes will change after the new branch has grown up. Computation formula of the value of morphactin concentration in other nodes will add the relevant node on the new branch, and remove the grown node, which basis on formula (1). The process will be repeated until there is no new branches growing.

\section{Nonlinear equations}

The general form of the nonlinear equations as:

$$
1\left\{\begin{array}{c}
f_{1}\left(x_{1}, x_{2}, \cdots, x_{n}\right)=0 \\
f_{2}\left(x_{1}, x_{2}, \cdots, x_{n}\right)=0 \\
\vdots \\
f_{n}\left(x_{1}, x_{2}, \cdots, x_{n}\right)=0
\end{array}\right.
$$

Solving the equations (1) is equivalent to solving the following extremal optimization problems:

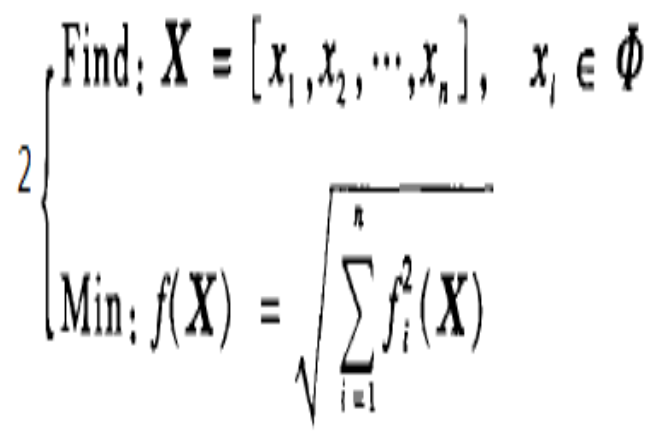


$\Phi$ is the solution interval equations (1), when the minimum value of $f(x)$ is 0 , the corresponding $\mathrm{x}$ is the solution of the equations.

\section{The analysis of simulation}

Select a typical nonlinear equations and a practical problem as an example to demonstrate.

Problem 1:

$$
3\left\{\begin{array}{l}
f_{1}(x)=x_{1}+\frac{x_{2}^{2} x_{4} x_{6}}{4}+0.75 \\
f_{2}(x)=x_{2}+0.405 \mathrm{e}^{1+x_{1} x_{2}}-1.405 \\
f_{3}(x)=x_{3}-\frac{x_{4} x_{6}}{2}+1.5 \\
f_{4}(x)=x_{4}-0.605 \mathrm{e}^{1-x_{3}^{2}}-0.395 \\
f_{5}(x)=x_{3}-\frac{x_{2} x_{6}}{2}+1.5 \\
f_{6}(x)=x_{6}-x_{1} x_{9}
\end{array}\right.
$$

Problem 2: I-shaped geometric properties of size

$$
{ }_{4}\left\{\begin{array}{l}
A=2 d b+d(h-2 d) \\
I_{y}=b h^{3} / 12-(b-d)(h-2 d)^{3} / 12 \\
I_{x}=d b^{3} / 6+(h-2 d) d^{3} / 12
\end{array}\right.
$$

The $b$ is the width of I-shaped beam, $h$ is the height of I-shaped beam , $d$ is the thickness of I-shaped beam . When A $=85, I_{y}=6874.79, I_{x}=179.79$, Document 4 gives a set of solutions of the equations (4).

The choice of ant colony algorithm, genetic algorithm and plant growth algorithm for 100 experiments on the above two problems randomly. Table 1 lists the success probability of the 3 different algorithms to solve two problems in 100 experiments. 
Plant growth algorithm on the two problem solving reliability reached to $100 \%$, significantly higher than ant colony algorithm and genetic algorithm.

Table 1 Comparison of convergence reliability

\begin{tabular}{|c|c|c|c|}
\hline \multirow{2}{*}{ problems } & \multicolumn{3}{|c|}{ algorithms } \\
\cline { 2 - 4 } & $\begin{array}{c}\text { ant colony } \\
\text { algorithm }\end{array}$ & genetic algorithm & $\begin{array}{c}\text { plant growth } \\
\text { algorithm }\end{array}$ \\
\hline Problem 1 & 30 & 31 & 100 \\
\hline Problem 2 & 49 & 50 & 100 \\
\hline
\end{tabular}

Table 2 Comparison of calculation cost

\begin{tabular}{|c|c|c|c|}
\hline \multirow{2}{*}{ problems } & \multicolumn{3}{|c|}{ algorithms } \\
\cline { 2 - 4 } & $\begin{array}{c}\text { ant colony } \\
\text { algorithm }\end{array}$ & genetic algorithm & $\begin{array}{c}\text { plant growth } \\
\text { algorithm }\end{array}$ \\
\hline Problem 1 & 310.6 & 309.5 & 233.4 \\
\hline Problem 2 & 291.3 & 290.4 & 212.5 \\
\hline
\end{tabular}

Seen from the data in Table 1 and Table 2, the convergence reliability and computational cost of plant growth algorithm is significantly better than ant colony algorithm and genetic algorithm, convergence speed and solution accuracy of plant growth algorithm is high.

\section{Conclusions}

This paper proposes a plant growth algorithm, the plant growth algorithm is applied to solve nonlinear equation group, the performance has been improved obviously, and as the problem size increases, the improvement is more obvious. Experiments show that, the new algorithm has strong global search ability, convergence speed and solution accuracy of plant growth algorithm is high.

\section{Acknowledgment}


This work is supported by Dean Fund of Gansu Normal College for Nationalities.

\section{References}

[1] David A Cox, John B Little, Donald B. • Using Algebric Geometry [M]. New York: Springer Verlag, 1998.

[2] Hentenryck P Van, McAllester D, Kapur D. Solving Polynomial Systems Using a Branch and Prune Approach[J],SIAM Journal on Numerical Analysis, 1997(2):797-927.

[3] Zhao Ji, Xu Wen-bo, Sun Ju. Solving Systems of Nonlinear Equations Using Quantum-behaved Particle Swarm Optimization[J]. Application Research of Compute, 2007(24):80-82.

[4] Yang Wan-an, Zeng An-ping. New method of solving complicated nonlinear equation group[J]. Computer Engineering and Applications, 2009(28):41-42.

[5] Li T,Wang C F ,et al.A global optimization bionics algorithm for solving integer Programming plant growth simulation algorithm[J] .Systems Engineering - Theory \& Practice ,2005,25(1) :76 - 85.

[6] Li T, Wang Z t. Application of plant growth simulation algorithm on solving facility location problem [J]. Systems Engineering - Theory \& Practice, 2008, (12):107-115

[7] Sui Yunkang, Zhao Wenzhong. Two programming solution method and application of nonlinear equations[J]. The Chinese Journal of computational mechanics, 2002, 19(2): 245 - 246.

[8] Krzyworzcka S. Extension of the Lanczos and CGS methods to systems of nonlinear equations[J].Journal of ComPutational and APPlied Mathematies ,1996,69(l):181-19 0. 